Secoud Meeting, December 10th, 1886.

George Thom, Esq President, in the Chair.

Composition de Mathématiques Élémentaires proposée au

Concours d' Agrégation de 1886.

Solution par M. Paul Avbert

On donne un cercle et deux points $P$ et $Q$ situes sur $u$. dixmetre, on joint les points $P$ et $Q$ aux extrénités $A$ et $B$ d'ur siarnètre du cercle par les druites $I^{\prime} A$ et $Q B$ qui se coupent ane point $M$. On fait tourner le diamitre $A B$ ct on demande

1. D'étudier les variations du rappo' ' $\frac{M A}{M B}$, et de construire la figure quand le rapport a une valeur donnéc.

II. $D^{\prime}$ étudier les variations de l' angle. $A M B$, et de construire la figure quand cat angle a ume valeur donnée.

III. $A^{\prime}$ et $B^{\prime}$ étant les seconds points d' intersection des droites $M A$, $M B$ avec la circonfórence donnée, trouver lo liene du contre du cercle circonscrit au triangle $M A^{\prime} B^{\prime}$.

I. On a (fig. 45)

$$
\frac{M A}{M B}=\frac{\sin A B M}{\sin B A M}
$$

Menons par le point $B$ la parallèle $B R$ a $M P$, on a

$$
\frac{\sin A B M}{\sin B A M}=\frac{\sin O B Q}{\sin O \overline{B R}}=\frac{O Q \sin B O Q}{B Q} \cdot \frac{O R \sin B O Q}{B R} \overline{O Q} \cdot \frac{B R}{B Q} .
$$

Appelons $a$ et $b$ les distances des points $P$ et $Q$ au centre de la circonférence donnée dont nous désignorons le rayon par $\mathrm{P}$, et soit $\lambda$ l' angle $B O Q$ du diamètre mobile $A B$ aveo le diametre fixe $P Q$ On a

$$
\frac{\mathrm{MA}}{\mathrm{MB}}=\frac{b}{a} \frac{\mathrm{BR}}{\mathrm{BQ}}=\frac{b}{a} \sqrt{\frac{a^{2}+\mathrm{R}^{2}-2 a \mathrm{R} \cos \lambda}{b^{2}+\mathrm{R}^{2}-2 b \operatorname{Rec} \lambda}}
$$

Si les points $P$ et $Q$, au lieu d' être de part et $d$ ' autre du point 0 , sont du miême côté de ce point (fig. 46), il est facile de voir que 
les relations précédentes subsistent, à condition de changer sous le radical $a$ en $-a$. On est donc amené à étudier les variations de l'expression

$$
y=\frac{a^{2}+\mathrm{R}^{2}-2 a \mathrm{R} \cos \lambda}{b^{2}+\mathrm{R}^{2}-2 b \mathrm{R} \cos \lambda}
$$

quand $\lambda$ varie de $0^{\circ}$ a $360^{\circ}$. Il suffira de faire varier $\lambda$ de $0^{\circ}$ à $180^{\circ}$, puisque $\cos \lambda$ prend toutes ses valeurs possibles dans cet intervalle, puis de prendre pour l' intervalle de $180^{\circ}$ á $360^{\circ}$ les valeurs de l' expression déjà trouvées mais dans l' ordre inverse.

Quand $\cos \lambda$ varie de $+1 a-1$ les deux termes de la fraction $y$ restent toujours positifs. Par suite $y$ ne devient jarnais nulle ni infinie. Supposons d' abord $P$ et $Q$ de part et d'autre du centre. On peut écrire

$$
\begin{aligned}
y & =\frac{a}{b} a\left(a^{2}+\mathrm{R}^{2}\right)-2 a b \mathrm{R} \cos \lambda \\
& =\frac{a}{b}\left[1+\frac{b\left(a^{2}+\mathrm{R}^{2}\right)-2 a b \mathrm{R} \cos \lambda}{a\left(b^{2}+\mathrm{R}^{2}\right)-2 a b \mathrm{R} \cos \lambda}\right] \\
& =\frac{a}{b}\left[1+\frac{\left(\mathrm{R}^{2}-a b\right)(b-a)}{a\left(b^{2}+\mathrm{R}^{2}\right)-2 a b \mathrm{R} \cos \lambda}\right]
\end{aligned}
$$

Quand $\cos \lambda$ varie de +1 a -1 le dénominateur de la fraction augmente constamment. Si son numérateur est positif, $y$ dininue; si ce numérateur est négatif, $y$ augmente continuellement. Le rapport $\frac{\mathrm{MA}}{\mathrm{MB}}$ varie de $\frac{b}{a} \frac{\mathrm{R}-a}{\mathrm{R}-b}$ à $\frac{b}{a} \frac{\mathrm{R}+a}{\mathrm{R}+b}$ toujours dans le même sens, la première expression étant prise en valcur absolue.

Si les points $\mathbf{P}$ et $\mathbf{Q}$ sont du même côté du centre, on a

$$
\frac{\mathrm{MA}}{\mathrm{MB}}=\frac{b}{a} \sqrt{\frac{a^{2}+\mathrm{R}^{2}+2 a \mathrm{R} \cos \lambda}{b^{2}+\mathrm{R}^{2}-2 b \mathrm{R} \cos \lambda}} .
$$

Quand $\cos \lambda$ varie de $+1 \mathbf{a}-1$, le numérateur de la fraction diminue, son dénominateur augmente. Done le rapport diminue continuellement. Il varie de

$$
\frac{b}{a} \frac{a+\mathrm{R}}{b-\mathrm{R}} \text { a } \frac{b}{a} \frac{a-\mathrm{R}}{b+\mathrm{R}}
$$

Dans les deux cas le rapport $\frac{\mathrm{MA}}{\mathrm{MB}}$ varie toujours dans un certain sens quand $\lambda$ eroit de $0^{\circ}$ a $180^{\circ}$, et dans le sens contraire quand $\lambda$ continue it croitre de $180^{\circ}$ a $360^{\circ}$.

Construction gémétrique: La construction da point Ml défend 
de la position du diamètre $A B$, qui est elle même déterminée par la position du point $B$. Or nous avons vu que

$$
\frac{\mathrm{BR}}{\mathrm{BQ}}=\frac{a}{b} \frac{\mathrm{MA}}{\overline{\mathrm{M}} \overline{\mathrm{B}}} \text {. }
$$

Si le rapport $\frac{M A}{\overline{M B}}$ eat donné, on connaît donc la valeur du rapport $\widetilde{\mathrm{BR}}$. Pour obtenir le point $\mathrm{B}$, on déterminera d' abord les deux points $I$ et $I^{\prime}$ du diamètre $Q R$ (fig. 47) dont le rapport des distances aux points $Q$ et $R$ a la valeur $\frac{a}{b} \frac{\mathrm{MA}}{\mathrm{MB}}$, puis on décrira sur II' comme diamètre une circonférence qui coupera la circonférence donnée en deux points répondant à la question, si la valeur donnée pour le rapport $\frac{\mathrm{MA}}{\mathrm{MB}}$ est comprise entre les valeurs limites trouvées précédemment. On peut retrouver ces valeurs limites en discutant les conditions de possibilité de la construction géométrique.

II. Étude de l' angle AMB.

On a dans le triangle $B Q R$ dont l' angle $B$ est egal à l'angle AMB

$$
\begin{aligned}
\text { d' où }^{2} & =\overline{\mathrm{Q}} \overline{\mathrm{Q}}^{2}+\overline{\mathrm{BR}}^{2}-2 \mathrm{BQ} \cdot \mathrm{BR} \cos \mathrm{B}, \\
\text { Mais } \quad \cos \mathrm{B} & =\frac{\overline{\mathrm{BQ}}^{2}+\overline{\mathrm{BR}}^{2}-\overline{\mathrm{QR}}^{2}}{2 \mathrm{BQ} \cdot \mathrm{BR}} . \\
\overline{\mathrm{BQ}}^{2} & =\mathrm{R}^{2}+b^{2}-2 b \mathrm{R} \cos \lambda, \\
\overline{\mathrm{BR}}^{2} & =\mathrm{R}^{2}+a^{2}-2 a \mathrm{R} \cos \lambda, \\
\overline{\mathrm{QR}}^{2} & =(a-b)^{2} .
\end{aligned}
$$

Effectuant ces substitutions, il vient

$$
\cos \mathrm{B}=\frac{\mathrm{R}^{2}+a b-(a+b) \mathrm{R} \cos \lambda}{\sqrt{\left(\mathrm{R}^{2}+b^{2}\right)\left(\overline{\mathrm{R}}^{2}+a^{2}\right)-2(a+b)\left(a b+\mathrm{R}^{2}\right) \mathrm{R} \cos \lambda+4 a b \mathrm{R}^{2} \cos ^{2} \lambda}} .
$$

Il est avantageux de se servir de l' expression de tgB. On trouve salis difficulté en partant de la formule précédente

$$
\operatorname{tg} \mathrm{B}=\frac{(a-b) \mathrm{R} \sin \lambda}{\mathrm{R}^{2}+a b-(a+b) \mathrm{R} \cos \lambda}
$$

Posons $\operatorname{tg} \frac{\lambda}{2}=t$, il vient

$$
\operatorname{tg} \mathrm{B}=\frac{2(a-b) \mathrm{R} t}{(\mathrm{R}+a)(\mathrm{R}+b) t^{2}+(\mathrm{R}-a)(\mathrm{R}-b)}
$$


Nous nous servirons de cette expression pour étudier les variations de l' angle $\mathrm{B}$ quand $\lambda$ varie de $0^{\circ}$ à $360^{\circ}$, c'est-ádire, quand $t$ varie de zéro à $+\infty$ et de $-\infty$ à zéro. Il suffit de faire varier $t$ de zéro à $+\infty$, car quand il varie de $-\infty$ à zéro, t $g$. $B$ reprend les mêmes valeurs dans l' ordre inverse mais changées de signe.

Pour cela considérons la fonctien $y=\frac{m t}{p t^{2}+q}$.

Si on écrit la relation qui lie $y$ à $t$ sous la forme

$$
p y t^{2}-m t+q y=0
$$

on voit qu' $y$ ne pourra prendre que les valeurs satisfaisant à l' inégalité

$$
m^{2}-4 p q y^{2}>0 \text {. }
$$

Deu $x$ cas ì distinguer $\quad 1^{\circ} p q<0$.

$L^{\prime}$ ' inégalité est toujours verifiee et $y$ peut prendre toutes les valeurs de $-\infty \mathrm{a}+\infty$.

Posons $p q=k^{2} \quad L$ inégalité s' écrit

$$
2^{\circ} p q>0 \text {. }
$$

$$
(m-2 k y)(m+2 k y)>0
$$

et, $m$ et $k$ designant des quautitís positives, on doit avoir

$$
-\frac{m}{2 k}<y<\frac{m}{2 k}
$$

Dans ce cas $y$ reste compris entre deux linites correspondant aux valeurs de $t$ fournies par la relation précédente, oi l'on donne successivement à $y$ les deux valeurs $-\frac{m}{2 k}$ et $+\frac{m}{2 k}$, et en prenant chaque fois pour $t$ la demi-somme des raciues de l' equation ainsi obtenue

$$
t=\frac{k}{p} \text { et } t=+\frac{k}{p} \text {. }
$$

En résumé, si nous ne considérons que les valeurs positives de $t$, les variations de $y$ corresnondantes sont représentées pour le premier cay par la courbe (fig. 48), et pour le second cas par la courbe (fig. 49), si on a $p>0$, et par la courbe (fig. 50) si on a $p<0$.

Revenons maintenant a l' élude de tg $B$ et appliquons les résultats que nous venons d' obtenir.

La condition relative au signe de $p q$ comeluit à étudier le signe de $(\mathrm{R}+a)(\mathrm{R}+b)(\mathrm{R}-a)(\mathrm{R}-b)$. Cette expression ne change pas quand on y remplace $a$ par $-a$. Done dans tous les cas de figure elle est du signe de $(\mathrm{R}-a)(\mathrm{R}-\mathrm{b})$.

$1^{\circ}(\mathrm{R}-a)(\mathrm{R}-b)<0$, ce qui exprime que l'un des points est 
intérieur et l' autre extéricur à la circonférence. Dans ce cas l' angle $\mathrm{B}$ varie de $180^{\circ}$ a $90^{\circ}$, puis diminue jusqu' à $0^{\circ}$; il est droit pour

$$
\operatorname{tg} \frac{\lambda}{2}=\sqrt{\frac{(a-R)(\mathrm{R}-b)}{(\mathrm{R}+a)(\mathrm{R}+b)}} \text { ou } \cos \lambda=\frac{\mathbf{R}^{2}+a b}{(a+b) \mathrm{R}},
$$

comme l' indiquait la voleur de $\cos \mathrm{B}$.

$2^{\circ}$. $(\mathrm{R}-a)(\mathrm{R}-b)>0$ Les deux points sont alors tous deux extérieurs on tous deux intérieurs.

Si on a $(R+a)(R+b)>0$, ce qui exclut le cas où les points seraient extérieurs et du même côté, l' angle $B$ est toujours aigu, il croît de zéro à une valeur maxima donné par

$$
\operatorname{tg} \mathrm{B}=\frac{(a-b) \mathrm{R}}{\sqrt{\left(\mathbf{R}^{2}-a^{2}\right)\left(\overline{\left.\mathbf{R}^{2}-b^{2}\right)}\right.}}
$$

et correspondante a $\quad \operatorname{tg} \frac{\lambda}{2}=\sqrt{\frac{(\overline{\mathrm{R}-a)}(\overline{\mathrm{R}-b)}}{(\overline{\mathrm{R}+a})(\mathrm{R}+b)}}$.

Si on a $(\mathrm{R}+a)(\mathrm{R}+b)<0$, ce qui exprime que les deux points sont extérieurs à la circonférence et du même côté, l' angle $B$ est toujours obtus, il varie de $180^{\circ}$ a un minimum pour augmenter ensuite jusqu' à $180^{\circ}$.

En rísumé, quand les points sont l' un intérieur l' autre extérieur a la circonférence, l'angle $B$ varie toujours dans le méme sens et prend toutes les valeurs de $0^{\circ}$ a $180^{\circ}$.

Quand les points sont tous deux intérieurs, ou tous deux extérieurs l' angle $B$ est toujours soit aigu soit obtus; dans l6 premier cas, il commence par croitre jusqu' à un certain maximum pour décroître ensuite jusqu' à zéro; dans le second cas, il décroít de $180^{\circ}$ à un winimum qu' il dépasse ensuite pour retourner à la valeur $180^{\circ}$.

Pour construire géométriquement le point $M$ répondant à une valeur donnée de l' angle $M$, il suffit de déterminer la position du point $B$. $\quad L$ ' angle $Q B R$ étant connu, et les points $Q$ et $R$ étant fixes, on décrira sur $R Q$ un segment de cercle capable de l' angle donné. Les points d' intersection de ce segment avec la circonférence donnée donnent les points $B$ chercliés. On voit que si les points sont l'un intérieur et l'autre ex térieur, il y aura toujours au-dessus du diamètre $Q R$ un point $\mathrm{B}$ répondant à la question. Si les points sont tous deux intérieurs ou tous deux extérieurs, il pourra y avoir deux positions, uno seule, ou aucune suivant les cas. L' étude des conditions de possibilité de cette construction géométrique du point $\mathrm{B}$ conduirait anx résultats établis plus haut par une autre voie. 
III. Les triangles semblables PMQ et RBQ donnent

on, puisque $B R=P A$,

$$
\frac{\mathrm{BR}}{\tilde{\mathrm{PM}}}=\frac{\mathrm{QR}}{\mathrm{QP}}=\frac{\mathrm{QB}}{\mathrm{QM}} \text {; }
$$

$$
\frac{\mathrm{PA}}{\mathrm{PM}}=\frac{\mathrm{QK}}{\mathrm{QP}} \text {. }
$$

Le dernier rapport est constant, puisque les points $Q$ et $R$ sont fixes. Le point $P$ étant fixe, il résulte de cette égalité que le point $M$ est sur une circonférence homothétique à la circonférence donnée par rapport au point $P$, qui est centre $d^{\prime}$ homothétie directe.

On a aussi $\frac{\mathrm{QB}}{\mathrm{QM}}=\frac{\mathrm{QR}}{\mathrm{Q} \overline{\mathbf{P}}}$.

Cette égalité montre que le point $Q$ est le centre d' homothétie inverse des deux circonférences.

Ces résultats se rapportent a la figure 45 ; ils seraient renversés pour le cas de la figure 46. Dans tous les cas $P$ et $Q$ sont les centres d' homothétie directe et inverse de la circonférence donnée $O$, et de la circonférence lieu du point $M$, que nous appellerons circonférence $O^{\prime}$. Considérons par exemple la figure 51. Les points $\mathbf{M}$ et $\mathbf{A}^{\prime}$ sont antihomolognes. La tangente en $A^{\prime}$ au cercle $O$ et la tangente en $M$ a la circonférence $\mathrm{O}^{\prime}$ font donc avec $\mathrm{MA}^{\prime}$ des angles égaux, et se coupent en I sur l' axe radical des deux cercles. Pour la même raison, les points $M$ et $B^{\prime}$ étant anti-homologues, la tangente en $B^{\prime}$ au cercle $O$ rencontre MI sur l' axe radical, c'est-d-dire au point $I$, et les angles IMB' et IB'M sont égaux. Le point I est donc situé sur les perpendiculaires élevées au milieu de $\mathrm{MA}^{\prime}$ 'et au milieu de $\mathrm{MB}^{\prime}$; c' est donc le centre du cercle circonscrit au triangle $M A^{\prime} B^{\prime}$. Il résulte des constructions précédentes que ce point est toujours sur l' axe radical des circonférences $\mathrm{O}$ et $\mathrm{O}^{\prime}$. Donc le lieu demandé est cet axe radical.

The Equilateral and the Equiangular Polygon.

By R. E. Allardicr, M.A.

\section{The Equilateral Polygon.}

Since an $n$-gon is determined by $2 n-3$ conciitions, anc $n-i$ conditions are involved in its being equilateral, there are still in the case of an equilateral $n$-gon $n-2$ conditions to be determined. These $n-2$ conditions cannot all be given in terms of the angles, since ar 